\title{
Utility of DXA screening for diagnosis of osteoporosis in US veterans aged 70 years and older
}

\author{
Joanna Khatib, ${ }^{1}$ Kim Stote, ${ }^{2}$ Aidar R Gosmanov ${ }^{1,3}$
}

${ }^{1}$ Department of Medicine, Albany Medical College, Albany, New York, USA ${ }^{2}$ Department of Nutrition, Health Sciences, SUNY, Empire State College, Albany, New York, USA ${ }^{3}$ Section of Endocrinology, Stratton VA Medical Center, Albany, New York, USA

\section{Correspondence to} Dr Aidar R Gosmanov, Section of Endocrinology, Stratton VA Medical Center, Albany, NY 12208, USA; aidar.gosmanov@va.gov

Accepted 28 August 2017 Published Online First 2 October 2017
Check for updates

To cite: Khatib J,

Stote K, Gosmanov AR.

$J$ Investig Med

2018;66:298-303.

\begin{abstract}
Dual energy $X$-ray absorptiometry (DXA) is

recommended for osteoporosis screening in men aged 70 years and older but supportive data is limited. The aim of this study was to determine the efficacy of DXA for the diagnosis of increased fragility in this group of subjects. We retrospectively identified men aged 70 years and older without prior history of fracture and/or conditions predisposing to low bone mineral density (BMD) who attended the VA endocrinology clinic and performed DXA for osteoporosis screening. We analyzed the relationship between BMD and demographic, anthropometric data and biochemical parameters using linear regression models. Out of 55 subjects identified, 13 (24\%) men had normal BMD, 30 $(54 \%)$ had osteopenia and $12(22 \%)$ had a diagnosis of osteoporosis based on the femoral neck (FN) T-score. Lumbar spine T-scores were normal in all three groups. Weight and body mass index (BMI) were significantly higher in the normal BMD group compared with the osteopenia and osteoporosis groups $(p<0.001)$. After adjustments for age, weight, BMI, vitamin D concentrations, and diabetes status, differences in the FN BMD among the groups remained significant $(p<0.001)$. Based on the Fracture Risk Assessment Tool (FRAX) score calculations in 43 non-osteoporotic patients, 15 patients with osteopenia had a 10-year hip fracture probability $\geq 3 \%$. Screening with DXA in male US veterans aged 70 years and older without known osteoporosis risk factors revealed that up to $50 \%$ of men may qualify for diagnostic workup to determine the etiology of low BMD and/or to meet criteria to initiate pharmacological therapy to reduce future fracture risk.
\end{abstract}

\section{INTRODUCTION}

Osteoporosis is one of the leading causes of morbidity and mortality in older women and men. ${ }^{1}$ With nearly 1.5 million American men over the age of 65 in the USA having osteoporosis, an older man has a $25 \%$ chance of having an osteoporotic fracture during his lifetime ${ }^{2}$ with the mortality rate associated with hip fractures, as well as vertebral and other major fractures, being higher in men than in women. ${ }^{34}$ Therefore, early diagnosis of bone loss in men

\section{Significance of this study}

What is already known about this subject?

- Dual energy X-ray absorptiometry (DXA) screening for osteoporosis in men over 70 years old without osteoporosis risk factors is recommended but underutilized.

- There is paucity of data on osteoporosis detection rates by DXA in this group of patients.

\section{What are the new findings?}

- One out five white male veterans aged 70 and older without osteoporosis risk factors have osteoporosis based on DXA findings.

- Fragility risk prediction using Fracture Risk Assessment Tool (FRAX) calculations demonstrated that almost one out of three veterans with bone mineral density in normal or osteopenia range meet criteria for fracture preventive treatments.

- FRAX calculations that included DXA femoral neck T-score value showed better future fracture prediction compared with FRAX without DXA information.

How might these results change the focus of research or clinical practice?

- Screening for osteoporosis with DXA in older men is an effective tool to identify those at high risk for fracture and, therefore, should be actively used in clinical practice. Future studies should include men from other racial and ethnic groups as well as test if fracture prevention strategies could reduce fracture burden in this group of subjects.

should become a priority to decrease risk of the osteoporotic fracture.

The International Society of Clinical Densitometry, the American College of Physicians and the Endocrine Society recommend screening for osteoporosis using dual energy X-ray absorptiometry (DXA) for men 70 years of age and older who have no known fracture risk factors. ${ }^{5-7}$ It is thought that for every SD reduction in bone mineral density (BMD), men have a relative risk of fracture that is equal to, or even greater 
than, the relative risk in women. ${ }^{8}$ Though DXA-based diagnosis of osteoporosis is associated with high fracture risk, the majority of fractures occur in patients whose BMD is positioned in osteopenic or even normal BMD ranges. In addition to the densitometric diagnosis of osteoporosis as a fracture prediction tool, the Fracture Risk Assessment Tool (FRAX) has gained popularity as a vehicle to estimate the 10-year probability of having a hip fracture and/or a major osteoporotic fracture (hip, clinical spine, proximal humerus, or forearm) for subjects between the ages of 40 and 90 years and a T-score above -2.5 . $^{9}$ The Endocrine Society recommends that men with the FRAX fracture risk probability $\geq 20 \%$ for major osteoporotic fracture or $\geq 3 \%$ for hip fracture should receive pharmacological therapy. ${ }^{7}$

Though 70 years as cut-off age to perform screening DXA in men may appear reasonable, data to substantiate benefits for early diagnosis of osteoporosis at this age is limited. Furthermore, the US Preventive Task Forces concluded that the current evidence is insufficient to assess the balance of benefits and harms of screening for osteoporosis in men. ${ }^{10}$ We hence performed study to describe the prevalence of DXA-based osteoporosis in male US veterans aged 70 years and above without known osteoporosis risk factors and history of prior fractures. We also used the calculated FRAX score in those subjects who were found to have a T-score above -2.5 to explore further utility of DXA study in those who were found to have normal BMD or osteopenia.

\section{MATERIALS AND METHODS Study subjects and setting}

We performed a retrospective chart analysis of male patients $\geq 70$ years of age, who were seen in the general endocrinology clinic staffed by a single provider (ARG) in Stratton VA Medical Center, Albany, New York, USA. All subjects underwent measurements of BMD using the same DXA scanner (DXA, Lunar, GE Healthcare) at the anteroposterior lumbar spine (L1-L4) (L-spine) and at the femoral neck (FN) as per standard protocol stated by the manufacturer. The study was conducted between January 1, 2016 and October 30, 2016 and single radiologist read all DXA images.

We excluded any men with prior diagnosis of osteoporosis or prior or current treatment for a fragility fracture, on steroid therapy, with a diagnosis of chronic kidney disease stages 3-5, with severe vitamin D deficiency, prior diagnosis of hyperthyroidism, primary or secondary hyperparathyroidism, hypogonadism or who were on testosterone replacement therapy, with history of malignancy, HIV, personal or parental history of fracture, and those who were receiving androgen deprivation therapy for prostate cancer. Patients above the age of 90 years were excluded as well, as the FRAX score does not calculate fracture risk for patients in this age group.

We collected the following patient information: age, weight, height, diabetes status, tobacco use and serum 25-OH vitamin D concentrations, calcium and creatinine. From the DXA reports, we extracted BMD values and T-scores for lumbar spine (L-spine) and both FNs. We used the University of Sheffield FRAX tool (https://www.sheffield.ac.uk/FRAX/tool.jspt) specific for the US population to calculate the risk of hip fracture and major osteoporotic fracture. The Institutional Review Board of the Stratton VA Medical Center, Albany, New York, USA approved the study.

\section{Data analysis}

We stratified patients into several groups based on the lowest T-score findings in any anatomical site using the WHO criteria: normal BMD (T-score $>-1.0$ ), osteopenia (T-score -1.0 to $<-2.5$ ), and osteoporosis (T-score $\leq-2.5$ ). We also divided patients into two groups based on their probability of experiencing hip fracture risk over the next 10 years: $<3.0 \%$ and $\geq 3 \%$. Then, clinical and laboratory values between the groups were analyzed by Student's t-test for continuous variables and Fisher's exact test for categorical variables. We developed univariate and multivariate linear regression models to determine the significance of the differences and $\mathrm{X}^{2}$ tests (or Fisher's exact tests when appropriate) for categorical variables. Average and standard errors of the separate groups were calculated as well. Univariate and multivariate linear regression models were employed to determine the significance of the difference between the groups. Data were analyzed using SAS V.9.4 (SAS Institute, Cary North Carolina, USA). A p-value of 0.05 was considered significant.

\section{RESULTS}

\section{The role of DXA in the diagnosis of osteoporosis}

Of 476 male veterans who attended the endocrinology clinic between January and October 2016, 143 underwent DXA and 55 patients met the study criteria, all of whom were White, alone. We identified three groups of the patients based on BMD findings. Table 1 compares demographic, laboratory, and radiological findings among the patients with normal BMD, osteopenia and osteoporosis. We relied on the diagnosis of low BMD corresponding to a T-score of -1.0 and below based on the worst FN BMD findings, given the high likelihood of falsely positive BMD measurements in the L-spine among the elderly as explained by the artifacts related to spine osteoarthritis and degenerative intervertebral disks. Thirty patients $(55 \%$ of all patients) were found to have osteopenia and 12 patients $(22 \%$ of all patients) had been diagnosed with osteoporosis for the first time. There were no differences in mean age and serum $25-\mathrm{OH}$ vitamin $\mathrm{D}$ concentrations among the groups, while the patients with low BMD weighed significantly less and had a lower BMI (table 1). Prevalence of tobacco use and diabetes status (table 1) and average serum calcium and creatinine levels were not different among all three groups (data not shown). Among patients with diabetes, prevalence of pioglitazone use was not different among the groups (one patient in the normal BMD group, two patients in the osteopenia group, and one patient in the osteoporosis group).

The BMD and T-scores in both the left and right FN were significantly lower in the osteopenia and osteoporosis groups compared with the normal BMD group in unadjusted analyses $(p<0.0001)$ (table 1). Adjustments for age, weight, BMI, vitamin D concentration, and diabetes status did not alter the between the groups differences in the FN DXA findings. Importantly, none of the patients had been diagnosed with osteopenia or osteoporosis based on L-spine BMD measurements. Though both BMD and T-scores in 
Table 1 Patient clinical characteristics

\begin{tabular}{|c|c|c|c|c|c|}
\hline & Normal BMD & Osteopenia & Osteoporosis & $\mathrm{p}$ Value univariate & $\begin{array}{l}\mathrm{p} \text { Value* } \\
\text { multivariate }\end{array}$ \\
\hline $\mathrm{n}$ & 13 & 30 & 12 & & \\
\hline Age (years) & $73.9 \pm 3.5$ & $76.7 \pm 5.9$ & $75.7 \pm 4.5$ & 0.29 & \\
\hline Body weight (kg) & $107.1 \pm 13.5$ & $82.9 \pm 11.9$ & $78.1 \pm 20.3$ & 0.002 & \\
\hline Height (cm) & $179.7 \pm 6.6$ & $176.5 \pm 6.5$ & $170.2 \pm 5.9$ & $<0.0001$ & \\
\hline Body mass index $\left(\mathrm{kg} / \mathrm{m}^{2}\right)$ & $33.4 \pm 5.4$ & $26.3 \pm 4.4$ & $26.9 \pm 6.1$ & $<0.001$ & \\
\hline $25-\mathrm{OH}$ vitamin $\mathrm{D}, \mathrm{ng} / \mathrm{mL}$ & $31.6 \pm 18.5$ & $27.5 \pm 8.2$ & $26.3 \pm 10.0$ & 0.46 & \\
\hline Diabetes mellitus (yes) & $43 \%$ & $41 \%$ & $16 \%$ & 0.27 & \\
\hline Current smoking (yes) & $15 \%$ & $10 \%$ & $17 \%$ & 0.46 & \\
\hline BMD FN, left & $1.14 \pm 0.14$ & $0.86 \pm 0.07$ & $0.71 \pm 0.07$ & $<0.0001$ & $<0.0001$ \\
\hline BMD FN, right & $1.11 \pm 0.14$ & $0.85 \pm 0.06$ & $1.71 \pm 0.06$ & $<0.0001$ & $<0.0001$ \\
\hline T-score L-spine & $2.25 \pm 1.53$ & $0.77 \pm 1.32$ & $-0.80 \pm 1.64$ & $<0.0001$ & 0.052 \\
\hline T-score FN, left & $0.67 \pm 1.12$ & $-1.63 \pm 0.53$ & $-2.70 \pm 0.48$ & $<0.0001$ & $<0.0001$ \\
\hline T-score FN, right & $0.69 \pm 1.18$ & $-1.66 \pm 0.48$ & $-2.73 \pm 0.42$ & $<0.0001$ & $<0.0001$ \\
\hline FRAX, MOF, \% & $4.9 \pm 1.5$ & $8.5 \pm 1.5$ & $13.0 \pm 2.6$ & $<0.0001$ & $<0.0001$ \\
\hline FRAX, hip fracture, $\%$ & $0.9 \pm 0.8$ & $3.1 \pm 1.1$ & $6.2 \pm 1.6$ & $<0.0001$ & $<0.0001$ \\
\hline FRAX w/o T-score, MOF, \% & $5.7 \pm 1.1$ & $7.6 \pm 1.6$ & $7.5 \pm 1.8$ & 0.0013 & 0.64 \\
\hline FRAX w/o T-score, hip fracture, $\%$ & $1.6 \pm 0.7$ & $3.0 \pm 1.5$ & $3.0 \pm 1.8$ & 0.01 & 0.18 \\
\hline
\end{tabular}

Values are mean \pm SE for continuous variables and percentages for categorical variables. p Values are based on Student's t-tests for continuous variables and Fisher's exact tests for categorical variables.

${ }^{*}$ Adjusted for age, weight, body mass index, vitamin D concentrations, and diabetes status.

$\mathrm{BMD}$, bone mineral density; FN, femoral neck; FRAX, fracture risk assessment tool; L-spine, lumbar spine; MOF, major osteoporosis-related fracture.

the L-spine tended to be lower in osteoporosis and osteopenia groups, after adjustments for patients' baseline characteristics, these differences were no longer statistically significant (table 1).

We also analyzed the FRAX score in each group in order to predict the 10-year risk of hip fracture or major osteoporotic fracture (MOF) (table 1). Using conventional FRAX calculations, which included DXA-based T-score values, the mean 10-year risk of sustaining MOF or hip fracture gradually increased among the groups correlating with BMD decline in both unadjusted and adjusted models $(p<0.0001)$. When we calculated the FRAX score using only patients' clinical characteristics and without factoring in the lowest FN T-scores, the differences in fracture risk were not significant after adjustments for the baseline characteristics $(p>0.05)$.

\section{The role of the FRAX calculation with and without a $\mathrm{T}$-score in predicting fragility fracture}

Although BMD findings consistent with diagnosis of osteoporosis can predict high fracture risk, the majority of fractures occur in patients with BMD findings in the osteopenia range. The FRAX tool is intended to aid providers in calculating fracture risk for those subjects whose T-scores do not meet the criteria for DXA-based diagnosis of osteoporosis. Because in our cohort none of the patients had high risk for future major osteoporotic fracture, we attempted to characterize patients based on their 10-year hip fracture probability. Using the traditional FRAX calculation that included information on the worst FN T-score, we identified that 27 patients had future hip fracture risk of $\geq 3.0 \%$ (table 2 ). Of note is that all patients with osteoporosis $(n=12)$ had been confirmed to have high fracture risk and 15 out of 30 patients with osteopenia were found to have a FRAX score $\geq 3.0 \%$. All patients within the normal BMD group had a FRAX score $<3.0 \%$. Patients with $\geq 3.0 \%$ hip fracture risk were older and had lower weight and BMI. The T-scores in the FN were significantly lower in the FRAX score $\geq 3.0 \%$ group $(p<0.0001)$, while the L-spine findings were similar between these two groups $(\mathrm{p}>0.05)$ (table 2$)$.

We then calculated FRAX scores without factoring in the FN T-scores generated during the DXA study (table 3). Using this approach, only 19 patients had been categorized by future hip fracture risk of $\geq 3.0 \%$. We also found that seven patients with DXA-based diagnosis of osteoporosis unexpectedly had been found as having a FRAX score of $<3.0 \%$. All patients with normal BMD had a FRAX score $<3.0 \%$. The differences in BMD or T-scores in either $\mathrm{L}$-spine or FN were no longer appreciated between these two groups after adjustment for baseline patient characteristics (table 3).

\section{DISCUSSION}

Current clinical practice recommendations suggest initiating DXA for osteoporosis screening in men aged 70 years and older. There is, however, little evidence to support or refute this age cut-off in men. In this study, we showed that implementing opportunistic DXA screening in men without known fracture risk factors can detect osteoporosis based on BMD in one out of five men. In addition, we demonstrated for the first time that following the calculation of the FRAX score that incorporated the FN BMD measurement, the number of patients meeting criteria for pharmacological therapy may reach up to $50 \%$. Based on these results, there is not only apparent benefit of performing DXA in men who are 70 years of age and older but there is also early evidence to consider osteoporosis screening in men at a younger age. 
Table 2 FRAX with T-score, hip fracture risk of $\geq 3 \%$

\begin{tabular}{|c|c|c|c|c|}
\hline & Risk< $<3 \%$ & Risk $\geq 3 \%$ & p Value univariate & p Value* multivariate \\
\hline$n$ & 28 & 27 & & \\
\hline Age (years) & $74.2 \pm 4.4$ & $77.3 \pm 5.4$ & 0.024 & \\
\hline Body weight (kg) & $95.8 \pm 17.7$ & $79.9 \pm 15.2$ & 0.001 & \\
\hline Height (cm) & $177.5 \pm 5.6$ & $174.3 \pm 8.1$ & 0.10 & \\
\hline Body mass index $\left(\mathrm{kg} / \mathrm{m}^{2}\right)$ & $30.1 \pm 6.4$ & $26.3 \pm 4.5$ & 0.014 & \\
\hline $25-\mathrm{OH}$ vitamin $\mathrm{D}, \mathrm{ng} / \mathrm{mL}$ & $30.0 \pm 14.8$ & $27.1 \pm 8.2$ & 0.37 & \\
\hline Diabetes mellitus (yes) & $46 \%$ & $26 \%$ & 0.094 & \\
\hline BMD L-spine & $1.41 \pm 0.21$ & $1.25 \pm 0.20$ & 0.006 & 0.34 \\
\hline BMD FN, left & $1.02 \pm 0.17$ & $0.78 \pm 0.09$ & $<0.0001$ & 0.0004 \\
\hline BMD FN, right & $1.01 \pm 0.15$ & $0.77 \pm 0.08$ & $<0.0001$ & $<0.0001$ \\
\hline T-score L-spine & $1.58 \pm 1.61$ & $0.06 \pm 1.57$ & $<0.001$ & 0.10 \\
\hline T-score FN, left & $-0.33 \pm 1.34$ & $-2.22 \pm 0.70$ & $<0.0001$ & $<0.001$ \\
\hline T-score FN, right & $-0.50 \pm 1.16$ & $-2.32 \pm 0.53$ & $<0.0001$ & $<0.0001$ \\
\hline FRAX, MOF, \% & $6.1 \pm 1.7$ & $11.1 \pm 2.4$ & $<0.0001$ & $<0.0001$ \\
\hline FRAX, hip fracture, $\%$ & $1.6 \pm 0.9$ & $4.9 \pm 1.7$ & $<0.0001$ & $<0.0001$ \\
\hline
\end{tabular}

Values are mean \pm SE $p$ Values are based on Student's t-tests for continuous variables and Fisher exact tests for categorical variables.

${ }^{*}$ Adjusted for age, weight, body mass index, vitamin D concentrations, and diabetes status.

BMD, bone mineral density; FN, femoral neck; FRAX, fracture risk assessment tool; L-spine, lumbar spine; MOF, major osteoporosis-related fracture.

Osteoporosis is an underdiagnosed and undertreated disease and, particularly in men, is associated with high risk of nursing home admissions. ${ }^{11}$ BMD measured by DXA is currently used for diagnosis of osteoporosis, as it correlates bone strength with future fracture risk. ${ }^{12}$ In the Osteoporotic Fractures in Men Study (MrOS), a prospective non-interventional cohort study that included 5414 men above the age of 65 years with no prior fracture history, subjects underwent BMD screening at baseline and then were followed for 9 years. This study showed that men who had a BMD T-score $\leq-1.5$ at baseline were more likely to develop osteoporosis later. ${ }^{13}$ In our study, $22 \%$ of men aged 70 years and older without known factors were for the first time diagnosed with osteoporosis based on FN BMD. Another important finding was that more than half of our cohort patients had osteopenia. Of note is the most recent publication of the outcomes of opportunistic DXA screening in 463 US male veterans without significant history of conditions predisposing to increased fragility that was conducted in the Western region of the USA. ${ }^{14}$ The investigators found presence of the FN-based diagnosis of osteopenia in $48.8 \%$ and osteoporosis in $20.5 \%$ of the veterans. These results are in agreement with the findings of low BMD prevalence in our cohort of the patients.

One retrospective cohort study examined the correlation between high-risk fractures identified using the FRAX score and BMD criteria in 2873 men from Manitoba, Canada. Results showed that $80 \%$ of the patients who had a highrisk FRAX score had at least one T-score in the osteoporotic range. ${ }^{15}$ Therefore, in the study patients with osteopenia we further assessed 10-year fragility risk by using the FRAX score. Our results confirmed these findings that all patients

Table 3 FRAX without T-score, hip fracture risk of $\geq 3 \%$

\begin{tabular}{|c|c|c|c|c|}
\hline & Risk<3\% & Risk $\geq 3 \%$ & p Value univariate & p Value* multivariate \\
\hline $\mathrm{n}$ & 36 & 19 & & \\
\hline Age (years) & $73.2 \pm 2.9$ & $80.5 \pm 5.0$ & $<0.0001$ & \\
\hline Body weight (kg) & $93.1 \pm 17.5$ & $78.3 \pm 15.8$ & 0.003 & \\
\hline Height (cm) & $175.7 \pm 6.5$ & $176.2 \pm 8.3$ & 0.81 & \\
\hline Body mass index $\left(\mathrm{kg} / \mathrm{m}^{2}\right)$ & $29.9 \pm 5.8$ & $25.2 \pm 4.7$ & 0.004 & \\
\hline $25-\mathrm{OH}$ vitamin $\mathrm{D}, \mathrm{ng} / \mathrm{mL}$ & $30.0 \pm 14.8$ & $27.1 \pm 8.2$ & 0.37 & \\
\hline Diabetes mellitus (yes) & $42 \%$ & $26 \%$ & 0.26 & \\
\hline BMD L-spine & $1.35 \pm 0.23$ & $1.29 \pm 0.19$ & 0.37 & 0.99 \\
\hline BMD FN, left & $0.94 \pm 0.20$ & $0.82 \pm 0.10$ & 0.011 & 0.56 \\
\hline BMD FN, right & $0.92 \pm 0.19$ & $0.92 \pm 0.09$ & 0.047 & 0.57 \\
\hline T-score L-spine & $1.04 \pm 1.82$ & $0.41 \pm 1.58$ & 0.21 & 0.79 \\
\hline T-score FN, left & $-0.94 \pm 1.59$ & $-1.94 \pm 0.76$ & 0.013 & 0.45 \\
\hline T-score FN, right & $-1.14 \pm 1.43$ & $-1.96 \pm 0.65$ & 0.027 & 0.82 \\
\hline FRAX, MOF, \% & $6.10 \pm 0.99$ & $8.9 \pm 1.32$ & $<0.0001$ & 0.18 \\
\hline FRAX, hip fracture, $\%$ & $1.71 \pm 0.64$ & $4.34 \pm 1.24$ & $<0.0001$ & 0.007 \\
\hline
\end{tabular}

Values are mean \pm SE. $p$ Values are based on Student's t-tests for continuous variables and Fisher's exact tests for categorical variables.

BMD, bone mineral density; FN, femoral neck; FRAX, fracture risk assessment tool; L-spine, lumbar spine; MOF, major osteoporosis-related fracture. 


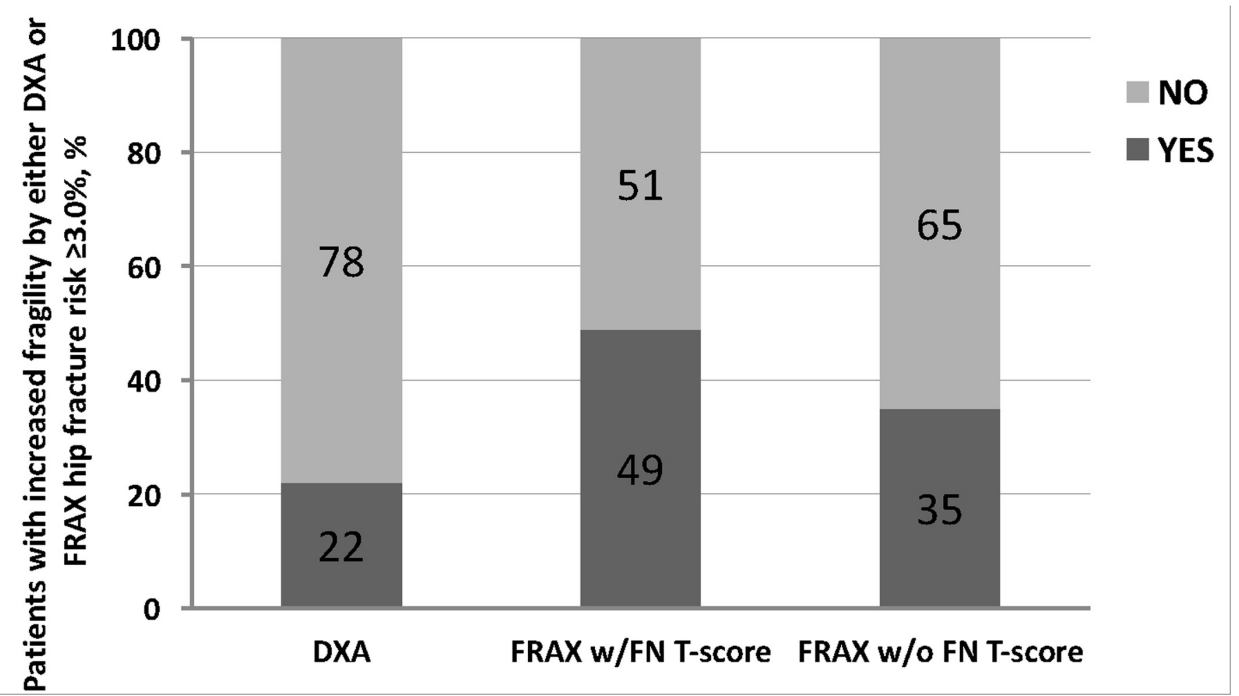

Figure 1 Comparison of fragility detection rates using dual energy X-ray absorptiometry (DXA) alone or by calculating 10-year hip fracture probability using fracture risk assessment tool (FRAX) with and without the femoral neck (FN) T-score.

with densitometric diagnosis of osteoporosis can be designated as having a high 10-year fracture risk. An another cohort demonstrated an increased accuracy of fracture prediction when BMD was combined with clinical risk factors in calculating fracture risk. ${ }^{16}$

In our study, when we combined the FRAX score with the BMD values, almost $50 \%$ of the population study did meet the criteria to initiate metabolic bone disease work up and/or treatment for osteoporosis. While the conventional FRAX score calculation involves incorporation of the DXA-derived value of the lowest FN BMD or T-score, some recently proposed to use FRAX without DXA data. Latter calculations were proposed to be useful in countries where DXA study availability is limited. ${ }^{17}$ However, when we omitted the T-score in the FRAX calculations, the 10-year fracture risk probability was less impressive suggesting the importance of FN BMD measurements in calculating the global fracture risk. In our cohort of the veterans, when we used the FRAX score without DXA-generated T-score, the proportion of the patients with a hip fracture risk $\geq 3 \%$ decreased to $35 \%$ (figure 1), while misclassifying 7 out of 12 men with DXA-proven osteoporosis as not meeting criteria for treatment. Our results suggest that combining the FRAX score with the FN T-score increases the sensitivity for detecting patients who should be evaluated for secondary causes and/or treated for osteoporosis.

The MrOS study showed that FN BMD correlates strongly with fracture risk, more so in men than in women. Though the spine BMD showed a strong correlation, it was weaker than the hip BMD. ${ }^{16}$ Our data suggest that in men 70 years of age and older, DXA evaluation of L-spine does not add to the detection of future fracture risk and its measurement can potentially be omitted during the DXA evaluation.

Limitations of our study include the retrospective design and assessment of only White, alone veterans in the Northeast region of the USA. A major strength of our study was utilizing electronic healthcare records.
This enabled us to exclude from the analyses patients with medical and endocrine conditions known to be associated with reduced bone mass. In addition, another strength was that a single radiologist analyzed the DXA reports. Future studies should include patients from multiple geographic regions of the USA and who belong to different ethnic groups as well as concurrently conduct basic biochemical evaluations to understand if there is common endocrine pathology(ies) present in men 70 years of age and older with new diagnosis of increased fragility.

\section{CONCLUSION}

Our data strongly argues for wider implementation of DXA for osteoporosis screening in men. Screening with DXA in male US veterans aged 70 years and older without known osteoporosis risk factors revealed that up to $50 \%$ of the patients would qualify for diagnostic workup to determine etiology of low BMD and/or therapy to reduce future fracture risk. We do recommend adding the FRAX score calculations to predict 10-year hip fracture risk in patients with DXA-based findings of FN osteopenia to augment the increased fragility risk case detection following screening DXA. It appears that in men aged 70 years and older using the FRAX score without the FN T-score is not adequate to determine future hip fracture risk.

Contributors JK researched data and prepared the manuscript. KS analyzed data and edited the manuscript. ARG is responsible for the study concept and design, researched data, and wrote and edited the manuscript. Authors would like to thank Jamila Benmoussa for helpful discussions. ARG is an employee of the US Department of Veterans Affairs and his opinions expressed in this mansucript are the author's personal opinions and do not necessarily represent the opinion of the Department of the Veterans Affairs.

Competing interests None declared.

Patient consent This was a retrospective chart analysis study.

Ethics approval Albany VAMC IRB, Albany NY.

Provenance and peer review Not commissioned; externally peer reviewed. 
(C) American Federation for Medical Research (unless otherwise stated in the text of the article) 2018. All rights reserved. No commercial use is permitted unless otherwise expressly granted.

\section{REFERENCES}

1 Johnell 0 , Kanis JA. An estimate of the worldwide prevalence and disability associated with osteoporotic fractures. Osteoporos Int 2006;17:1726-33.

2 Nguyen TV, Eisman JA, Kelly PJ, et al. Risk factors for osteoporotic fractures in elderly men. Am J Epidemiol 1996;144:255-63.

3 Bliuc D, Nguyen ND, Milch VE, et al. Mortality risk associated with low-trauma osteoporotic fracture and subsequent fracture in men and women. JAMA 2009;301:513-21.

4 Center JR, Nguyen TV, Schneider D, et al. Mortality after all major types of osteoporotic fracture in men and women: an observational study. Lancet 1999;353:878-82.

5 Qaseem A, Snow V, Shekelle P, et al. Screening for osteoporosis in men: a clinical practice guideline from the American College of Physicians. Ann Intern Med 2008;148:680-4.

6 Schousboe JT, Shepherd JA, Bilezikian JP, et al. Executive summary of the 2013 International Society for Clinical Densitometry Position Development Conference on Bone Densitometry. J Clin Densitom 2013;16:455-66.

7 Watts NB, Adler RA, Bilezikian JP, et al. Osteoporosis in men: an endocrine society clinical practice guideline. J Clin Endocrinol Metab 2012;97:1802-22.
8 Cummings SR, Cawthon PM, Ensrud KE, et al. BMD and risk of hip and nonvertebral fractures in older men: a prospective study and comparison with older women. J Bone Miner Res 2006;21:1550-6.

9 Kanis JA, Oden A, Johnell O, et al. The use of clinical risk factors enhances the performance of BMD in the prediction of hip and osteoporotic fractures in men and women. Osteoporos Int 2007;18:1033-46.

10 U.S Preventive Services Task Force. Screening for osteoporosis: U.S. preventive services task force recommendation statement. Ann Int Med 2011;154:356-64.

11 Rapp K, Rothenbacher D, Magaziner J, et al. Risk of nursing home admission after femoral fracture compared with stroke, myocardial infarction, and pneumonia. J Am Med Dir Assoc 2015;16:e7-12.

12 Cosman F, de Beur SJ, LeBoff MS, et al. Clinician's guide to prevention and treatment of osteoporosis. Osteoporos Int 2014;25:2359-81.

13 Gourlay ML, Overman RA, Fine JP, et al. Time to osteoporosis and major fracture in older men: the MrOS study. Am J Prev Med 2016;50:727-36.

14 Williams ST, Lawrence PT, Miller KL, et al. A comparison of electronic and manual fracture risk assessment tools in screening elderly male US veterans at risk for osteoporosis. Osteoporos Int 2017;28:3107-11.

15 Leslie WD, Majumdar SR, Lix LM, et al. High fracture probability with FRAX usually indicates densitometric osteoporosis: implications for clinical practice. Osteoporos Int 2012;23:391-7.

16 Leslie WD, Lix LM, Tsang JF, et al. Single-site vs multisite bone density measurement for fracture prediction. Arch Intern Med 2007;167:1641-7.

17 Kanis JA, McCloskey E, Johansson $\mathrm{H}$, et al. FRAX(R) with and without bone mineral density. Calcif Tissue Int 2012;90:1-13. 